\title{
SAMPLING PIECEWISE SMOOTH SIGNALS AND ITS APPLICATION TO IMAGE UP-SAMPLING
}

\author{
Xiaoyao Wei and Pier Luigi Dragotti \\ Imperial College London, UK
}

\begin{abstract}
In this paper we consider the problem of sampling piecewise smooth signals. The classical sampling theory is not able to sample them efficiently since the signals are neither bandlimited nor live in a shiftinvariant subspace. We propose a sampling scheme which still use the set of samples from a classical sampling set-up with an arbitrary acquisition device but achieves accurate reconstruction by making use of the non-linear approximate FRI reconstruction method in addition to the classical linear reconstruction. Specifically, we see the class of piecewise smooth signal as the sum of a piecewise polynomial, which is a signal that is fully specified by finite number of parameters and can be recovered with FRI non-linear methods, and a globally smooth term, which can be accurately recovered by linear reconstruction. We also show that our proposed scheme based on combining linear and non-linear reconstruction methods can be employed for resolution enhancement of discrete-time piecewise smooth signals and images.
\end{abstract}

\section{INTRODUCTION}

We consider the problem of sampling classes of piecewise smooth signals with the sampling set-up of Fig.1. Given the samples $y_{n}=\langle x(t), \varphi(t / T-n)\rangle$ taken with the acquisition device $h(t)=\varphi(-t / T)$, our goal is to recover the input signal $x(t)$ from $y_{n}$.

$x(t)$

$$
h(t)=\varphi(-t / T)
$$$$
x^{T}
$$

Fig. 1: Sampling set-up. Here $x(t)$ is the input signal, $h(t)$ is the impulse response of the acquisition device and $T$ is the sampling period. The samples are given by $y_{n}=\langle x(t), \varphi(t / T-n)\rangle$.

The classical answer to the sampling problem is given by the famous Shannon's theorem for sampling bandlimited signals. In that context, the reconstruction process is linear and the sampling and reconstruction kernel is the sinc function. Moreover, the sampling and reconstruction process is equivalent to projecting the input signal into the shift-invariant subspace of bandlimited signals and only this projection can be recovered. This framework has then been extended to classes of non-bandlimited signals that belongs to shift-invariant subspaces [1].

Piecewise smooth signals are neither bandlimited nor belong to a shift-invariant subspace. In this paper we model them as the sum of a piecewise polynomial signal and a globally smooth term (see Fig. 2).

This work is supported by the European Research Council (ERC) starting investigator award Nr. 277800 (RecoSamp).

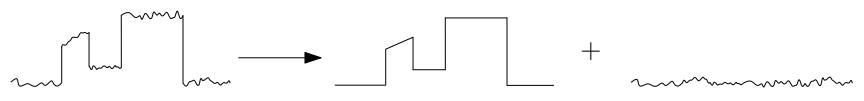

Fig. 2: A piecewise smooth signal can be modelled as the sum of a piecewise polynomial signal and a globally smooth signal.

Specifically if the smooth residual lives in a shift-invariant subspace generated by integer shifts of $\varphi(t / T)$, the piecewise smooth function can be expressed as:

$$
\begin{aligned}
x(t) & =\underbrace{\sum_{k=0}^{K-1} \sum_{r=0}^{R-1} a_{k, r}\left(t-t_{k}\right)_{+}^{r}}_{p(t)} \\
& +\underbrace{\sum_{n} \underbrace{\langle r(t), \tilde{\varphi}(t / T-n)\rangle}_{b_{n}} \varphi(t / T-n)}_{r(t)},
\end{aligned}
$$

where $t_{+}^{r}=\max (t, 0)^{r}$ and $\tilde{\varphi}(t)$ is the dual of $\varphi(t)$. Under this model, the globally smooth signal can be fully recovered using the traditional linear reconstruction method if $h(t)=\tilde{\varphi}(-t / T)$. The more recent theory of sampling signals with finite rate of innovation (FRI) $[2,3,4,5,6,7,8]$ can instead be used to reconstruct the piecewise polynomial term. The FRI framework employs non-linear reconstruction methods (i.e. Prony's method) but also requires the use of specific sampling kernel which are normally different from $\tilde{\varphi}(t)$. Due to this restriction it has not been possible, so far, to sample and accurately reconstruct piecewise smooth signals using a single acquisition device as in Fig. 1.

In this paper we solve this problem by using the approximate Strang-Fix methodology developed in [9] which has shown that FRI signals can be reconstructed approximately using arbitrary sampling kernels. This advance in FRI theory gives us the insight that we can have a sampling scheme which takes one set of samples with an arbitrary acquisition device as in the classical sampling set-up but in addition to the classical linear reconstruction, which would perfectly recover the smooth part, it takes advantage of the non-linear approximate FRI method to recover the piecewise polynomial part.

We then extend this idea to the case of 1-D and 2-D discretetime piecewise smooth signals where for the sake of simplicity, we assume $\varphi(t)$ and $\tilde{\varphi}(t)$ are scaling functions of a wavelet decomposition $[10,11]$. In this context we use the proposed hybrid reconstruction approach to enhance the resolution of the discrete-time signals. In the case of 2-D signals this leads to a new image up-sampling approach.

The paper is organized as follows. In Section 2 we explain how approximate Strang-Fix method in FRI enables sampling piecewise polynomials with an arbitrary acquisition device and how we combine this result with the classical linear reconstruction to sample 
piecewise smooth functions. In Section 3, we show how the idea in sampling continuous piecewise smooth functions can be employed to enhance the resolution of 1-D and 2-D discrete-time signals. We then show simulation results in Section 4 and conclude in Section 5.

\section{SAMPLING PIECEWISE SMOOTH FUNCTIONS}

In this part we first briefly introduce the non-linear reconstruction of the piecewise polynomial signals using FRI theory [3] where we emphasize that the approximate Strang-Fix method in FRI enables such reconstruction from samples taken with arbitrary sampling kernels [9]. Then we show how we use this result together with the linear reconstruction method to sample piecewise smooth signals.

Since the samples obtained by sampling a piecewise polynomial signal $p(t)$ with pieces of maximum degree $R-1(R>0)$, can be related to that of $R$-th derivative of $p(t)$, which is denoted by $p^{(R)}(t)$ and is a stream of (differentiated) Diracs, we concentrate on the sampling problem of streams of differentiated Diracs. Then this result can be used in the problem of sampling piecewise polynomials which interests us.

Consider a stream of differentiated Diracs:

$$
p^{(R)}(t)=\sum_{k=0}^{K-1} \sum_{r=0}^{R-1} b_{k, r} \delta^{(r)}\left(t-t_{k}\right) .
$$

Assume that $p^{(R)}(t)$ is sampled with a kernel $\varphi(t)$ that can reproduce exponentials $\mathrm{e}^{\alpha_{m} t}=\mathrm{e}^{m^{\prime} \lambda t}$ with $m^{\prime}=m-(M+1) / 2$ and $m=0, \ldots, M$. That is:

$$
\sum_{n \in \mathbb{Z}} c_{m, n} \varphi(t / T-n)=\mathrm{e}^{\alpha_{m} t / T} \quad \text { with } m=0,1, \ldots, M
$$

for a proper choice of the coefficients $c_{m, n}$. It is possible to show that a function satisfies (3) if and only if it meets the generalised Strang-Fix conditions:

$$
\hat{\varphi}\left(\alpha_{m}\right) \neq 0 \text { and } \hat{\varphi}\left(\alpha_{m}+j 2 \pi l\right)=0 \quad l \in \mathbb{Z} \backslash\{0\}
$$

where $\hat{\varphi}(s)$ is the bilateral Laplace transform of $\varphi(t)$.

Denote with $y_{n}=\left\langle p^{(R)}(t), \varphi(t / T-n)\right\rangle$ the observed samples. Consider now the following weighted sum of the samples, where the weights $c_{m, n}$ are those in (3) that reproduce $\mathrm{e}^{\alpha_{m} t / T}$, we have:

$$
\begin{aligned}
\tau_{m} & =\sum_{n} c_{m, n} y_{n}=\int_{-\infty}^{\infty} p^{(R)}(t) \mathrm{e}^{\alpha_{m} t / T} d t \\
& =\int_{-\infty}^{\infty} \sum_{k=0}^{K-1} \sum_{r=0}^{R-1} a_{k, r} \delta^{(r)}\left(t-t_{k}\right) \mathrm{e}^{\alpha_{m} t / T} d t \\
& =\sum_{k=0}^{K-1} \sum_{r=0}^{R-1} \hat{b}_{k, r}\left(m^{\prime}\right)^{r} u_{k}^{m^{\prime}}, \quad \begin{array}{l}
m=0,1, \ldots, M \text { and } \\
m^{\prime}=m-(M+1) / 2,
\end{array}
\end{aligned}
$$

where $\hat{b}_{k, r}=(-\lambda / T)^{r} b_{k, r}$ and $u_{k}=\mathrm{e}^{\lambda t_{k} / T}$.

The $t_{k}$ 's can be retrieved from $\tau_{m}$ using Prony's method (annihilating filter method). The key is to note that given a filter $\left\{h_{m}\right\}_{m=0}^{K}$ whose roots of its $z$-transform is:

$$
H(z)=\sum_{m=0}^{K R} h_{m} z^{-m}=\prod_{k=0}^{K-1}\left(1-u_{k} z^{-1}\right)^{R},
$$

then this filter can annihilate the sequence $\tau_{m}$. The $K R$ unknown coefficients of $h_{m}$ can be found by solving a Yule-Walker system constructed from at least $2 K R$ consecutive $\tau_{m}$. From the roots of the annihilating filter $h_{m}$ we obtain the locations $t_{k}$. Then the amplitudes can be found by solving, for example, the first $K R$ equations in (5).

Now regarding the problem of sampling piecewise polynomials, it can be shown that the $R$ th finite difference of $y_{n}$ is equivalent to the set of samples obtained by sampling $p^{(R)}(t)$ with the new kernel $\varphi(t) * \beta_{R-1}(t)$, where $\beta_{R-1}(t)$ is the B-spline of degree $R-1$ and this new kernel still meets the Strang-Fix conditions. Therefore we are able to use the result of sampling stream of differentiated Diracs for sampling piecewise polynomials.

When $\varphi(t)$ is an exponential reproducing kernel we can achieve exact reconstruction. For any other kernels $\varphi(t)$ which does not satisfy the generalized Strang-Fix condition we want to find a linear combination of $\varphi(t)$ with its shifted versions that provides the best approximation to a specific exponential. More precisely, we want to find coefficients $c_{n}$ such that:

$$
\sum_{n \in \mathbb{Z}} c_{n} \varphi(t-n) \approx \mathrm{e}^{\alpha t}
$$

For the sake of clarity, we use $c_{n}=c_{0} \mathrm{e}^{\alpha n}$ and then we can show that the error in approximating the exponential is [9]:

$$
\epsilon_{\text {approx }}(t)=\mathrm{e}^{\alpha t}\left[1-c_{0} \sum_{l \in \mathbb{Z}} \hat{\varphi}(\alpha+j 2 \pi l) \mathrm{e}^{j 2 \pi l t}\right] .
$$

Note that if the Laplace transform of $\varphi(t)$ decays sufficiently quickly, we can assume the terms $\hat{\varphi}(\alpha+j 2 \pi l)$ are close to zero for $l \in \mathbb{Z} \backslash\{0\}$. In this case, the approximation error is minimised when $c_{n}=\hat{\varphi}(\alpha)^{-1} \mathrm{e}^{\alpha n}$, requiring only the knowledge of $\varphi(t)$ at $\alpha$.

Using the approximate reproduction formula of (7) we are now able to reconstruct approximately piecewise polynomial signals using any kernel $\varphi(t)$. This fact leads to the sampling and reconstruction strategy for piecewise smooth signals highlighted in Fig. 3.

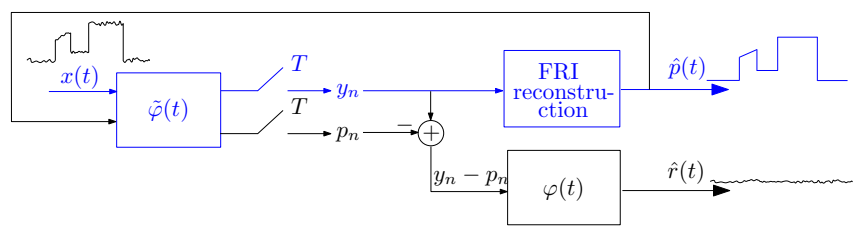

Fig. 3: Schematic diagram of our proposed sampling and reconstruction strategy for piecewise smooth signals.

Our proposed scheme first reconstructs the piecewise polynomial part $p(t)$ using the approximate Strang-Fix theory from the samples of the piecewise smooth function by treating the globally smooth residual as noise (the blue part in Fig. 3). Then the contribution from the piecewise polynomial part can be removed from the samples $y_{n}$ and the smooth residual $r(t)$ can be reconstructed by classical linear method (the black part in Fig. 3).

\section{APPLICATION TO UPSAMPLING 1-D PIECEWISE SMOOTH SIGNALS AND TO IMAGE UPSAMPLING}

In this section, we show that the idea of combining non-linear and linear reconstruction can be used also for enhancing the resolution of discrete-time signals. We first explain our proposed method to upsample 1-D signal using a wavelet-based scheme then its extension to image upsampling. 


\subsection{1-D Signal Upsampling}

We assume our samples $y_{0}[n]$ to be the lowpass output of a $J$-level wavelet transform applied to the high-resolution piecewise smooth signal $x[n]$ (see Fig. 4). Given the low-pass coefficients $y_{0}[n]$ the reconstruction stage gives us a coarse approximation of $x[n]$. Note that if we had access to the low-pass coefficients and also the wavelet coefficients, we could reconstruct the signal perfectly. Therefore to obtain a faithful upsampling result our goal is to estimate the bandpass and high-pass coefficients.

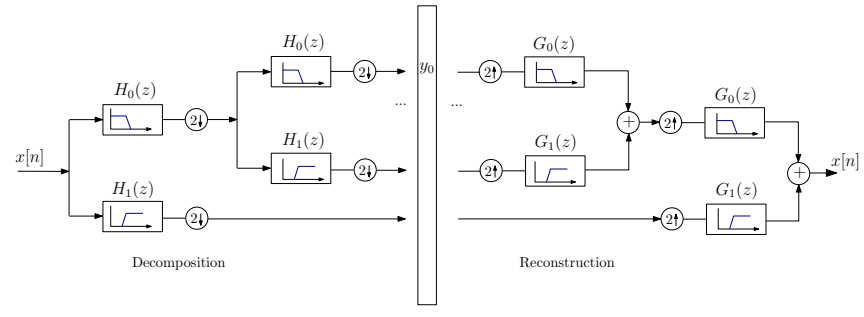

Fig. 4: $J$-level wavelet decomposition and reconstruction

Knowing the low-pass samples $y_{0}[n]$ and the specific low-pass filter used, we are able to reconstruct a piecewise polynomial function, called $\hat{x}_{\mathrm{FRI}}[n]$ by non-linear method based on the approximate FRI method introduced in Sec. 2. Then we recover the signal to its original resolution by keeping the coarse approximation and filling in the details given by feeding $\hat{x}_{\mathrm{FRI}}[n]$ into the other channels.

\subsection{Image Up-sampling}

Since scan-lines taken from images can be modelled as piecewise smooth functions, in this part we extend the method for upsampling 1-D signals to upsampling 2-D image by treating the two coordinates of 2-D images separately.

Assume we have access to a low-resolution image $y_{0}$ of size $N \times$ $N$, which is the low-pass version of a $J$-level $2 \mathrm{D}$ wavelet transform applied to the high-resolution image $x$ of size $2^{J} N \times 2^{J} N$.

We first interpolate the low-resolution image $y_{0}$ vertically to a image of size $2^{J} N \times N$ using the synthesis filter for coarse approximation. Then from each line of samples of length $N$ we reconstruct a piecewise polynomial of length $2^{J} N$ by FRI, in this way we get an image $\hat{x}_{\mathrm{FRI}_{x}}$ of size $2^{J} N \times 2^{J} N$ which contains horizontal edges. Similarly, we operate on the other coordinate of $y_{0}$ to recover an image $\hat{x}_{\mathrm{FRI}_{y}}$ of size $2^{J} N \times 2^{J} N$ which contains vertical edges.

Then we can finalize the reconstruction by inputting $\hat{x}_{\mathrm{FRI}_{x}}$ to the channels that capture horizontal edges and $\hat{x}_{\mathrm{FRI}_{y}}$ to the channels that capture vertical edges and either one of them to the channels that capture diagonal edges. We then diagonally operate downsampling and FRI upsampling to reduce the jaggies. Finally, the low-pass coefficients of our reconstructed high-resolution image are replaced with $y_{0}$ to ensure consistency.

\section{SIMULATION RESULTS}

\subsection{Sampling Piecewise Smooth Signals}

In this part, we show the reconstruction of a continuous-time piecewise smooth signal from samples $y_{n}=\langle x(t), \tilde{\varphi}(t / T-n)\rangle$ using our proposed scheme introduced in Sec. 2. Here $\varphi(t)$ is a spline and $x(t)$ is a combination of a piecewise constant function and a smooth function that lives in the shift-invariant subspace $V=$ $\operatorname{span}\{\varphi(t / T-n)\}_{n \in \mathbb{Z}}$. Fig. 5 demonstrates the reconstruction result and its comparison to the classical linear reconstruction. It indicates that our proposed method estimates the discontinuities of $x(t)$ accurately and therefore leads to substantial gain compared to the linear reconstruction.

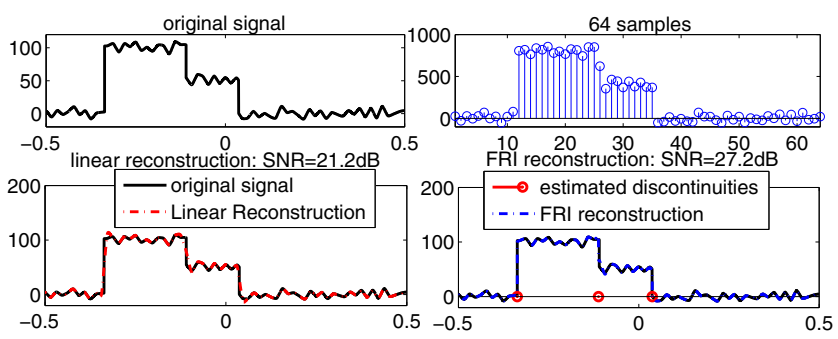

Fig. 5: Sampling a piecewise smooth function $x(t)$ with discontinuities $t_{k} \in[0,1)$ at rate $1 / T=1 / 64$. In this example, the classical linear method gives a reconstruction with $\mathrm{SNR}=21.2 \mathrm{~dB}$, and our proposed method yields a reconstruction with $\mathrm{SNR}=27.2 \mathrm{~dB}$.

\subsection{1-D Piecewise Smooth Signal Upsampling}

In the following simulations, we show the resolution enhancement results using our novel hybrid reconstruction method introduced in Sec. 3. In this section we assume our samples are the low-pass coefficients of 2-level wavelet decomposition applied to a highresolution piecewise smooth signal using biorthogonal 4.4 filter and we want to recover it to its original resolution. The original high resolution signal in this simulation is an image scan-line. (see Fig. 6). The upsampling result by our proposed method and its comparison to the linear reconstruction is shown in Fig. 7.
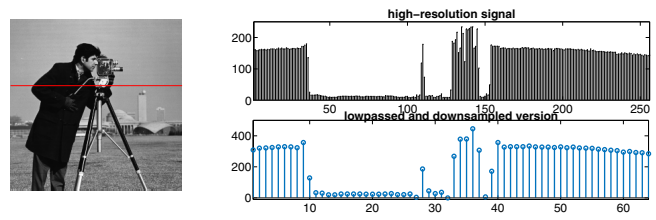

(a) a scan-line from (b) the scan-line is low-passed and a natural image. downsampled by 4 .

Fig. 6: The samples are the low-pass coefficients of 2-level wavelet decomposition applied to an image scan-line.

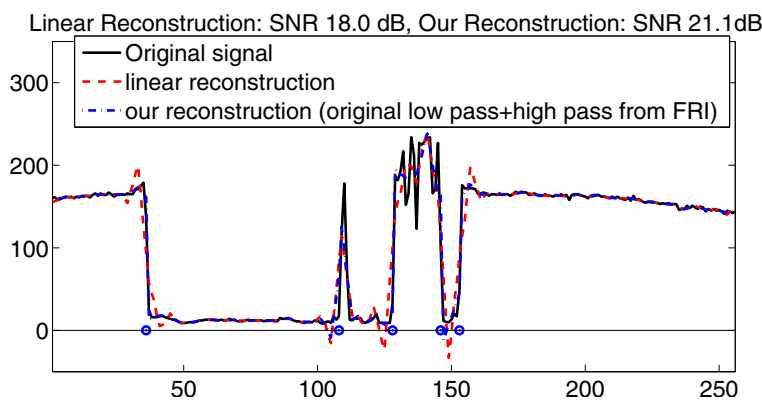

Fig. 7: Upsampling the low-resolution samples in Fig. 6 to its original resolution. The classical linear method yields a reconstruction with $\mathrm{SNR}=18.3 \mathrm{~dB}$, and our proposed method yields a reconstruction with $\mathrm{SNR}=20.8 \mathrm{~dB}$. 
The example in Fig.7 gives a clear indication of the gain added to the classical linear reconstruction method by adding the high frequency information obtained by non-linear reconstruction based on approximate FRI method.

\subsection{Image Upsampling}

In this part, the low-resolution images are obtained by downsampling the original images by a factor 4 using the 2-level 2D wavelet decomposition with biorthogonal 4.4 filter. We first test our proposed upsampling algorithm on a piecewise smooth image using a $2 \mathrm{D}$ extension of (1). That is, an image that is the sum of a $2 \mathrm{D}$ piecewise polynomial signal and a smooth $2 \mathrm{D}$ signal that can be perfectly recovered by the linear reconstruction. The comparison between our upsampling result and the linear reconstruction is shown in Fig. 8. It is evident that we gain by adding our estimated high-frequency information. Moreover, our result significantly outperforms several state-of-the-art upsampling methods as shown in Table 1 row 2.
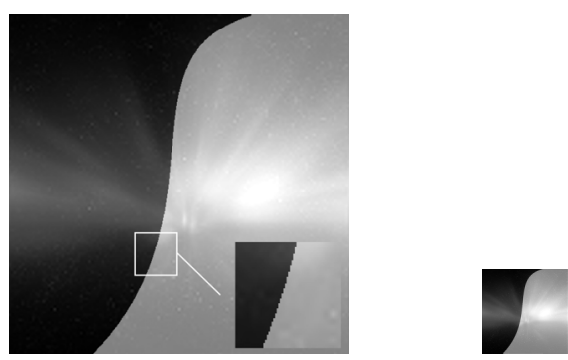

(a) original image

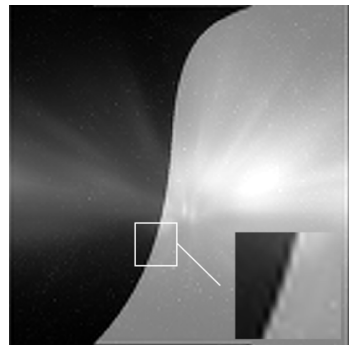

(b) low resolution image

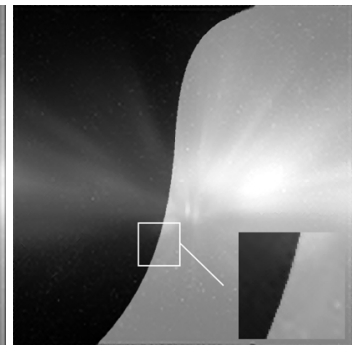

(c) linear reconstruction, (d) our reconstruction, $\mathrm{PSNR}=36.49 \mathrm{~dB}$ $\mathrm{PSNR}=42.81 \mathrm{~dB}$

Fig. 8: Upsampling results of a piecewise smooth image by linear reconstruction and by proposed method.

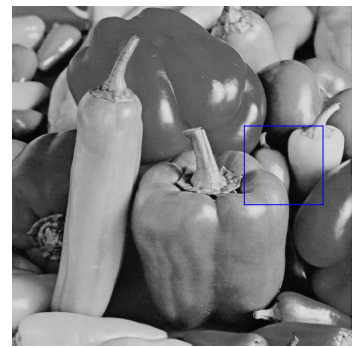

(a) original image

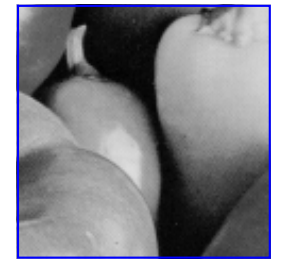

(a) the original image

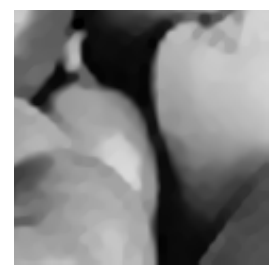

(d) total variation (e) minimization $\mathrm{PSNR}=30.98 \mathrm{~dB}$

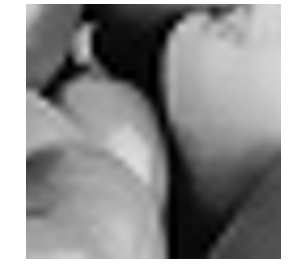

(b) linear reconstruction PSNR $=30.05 \mathrm{~dB}$

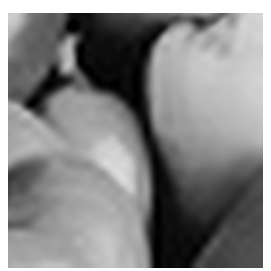

contourlet (f)

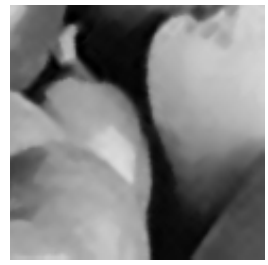

(c) our reconstruction $\mathrm{PSNR}=31.33 \mathrm{~dB}$

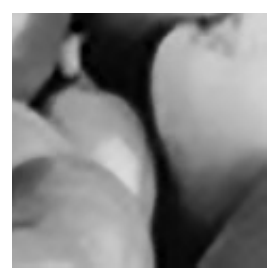

[13] method $\mathrm{PSNR}=31.02 \mathrm{~dB}$
Fig. 10: Upsampling results of Peppers (see Fig. 9) by different methods. Only cropped versions are shown for clarity.

Table 1: Comparisons of upsampling results given by different methods in terms of PSNR.

\begin{tabular}{|c|c|c|c|c|}
\hline PSNR & $\begin{array}{ll}\text { wavelet } & \text { our } \\
\text { linear } & \text { method }\end{array}$ & $\begin{array}{l}\operatorname{minimiz} \\
\mathrm{TV}[12]\end{array}$ & $\begin{array}{l}\text { con- } \\
\text { tourlet }[1 \text { ? }\end{array}$ & $\begin{array}{l}\text { dictionary } \\
\text { 3]learn- } \\
\text { ing [14] }\end{array}$ \\
\hline $\begin{array}{l}\text { piecewise } \\
\text { smooth }\end{array}$ & $36.49 \mathrm{~dB} 42.81 \mathrm{~dB}$ & $39.97 \mathrm{~dB}$ & $37.01 \mathrm{~dB}$ & $38.78 \mathrm{~dB}$ \\
\hline Peppers & $30.05 \mathrm{~dB} 31.33 \mathrm{~dB}$ & $30.98 \mathrm{~dB}$ & $30.34 \mathrm{~dB}$ & $31.02 \mathrm{~dB}$ \\
\hline Lena & $29.38 \mathrm{~dB} 30.11 \mathrm{~dB}$ & $29.92 \mathrm{~dB}$ & $29.73 \mathrm{~dB}$ & $29.96 \mathrm{~dB}$ \\
\hline Cameraman & $28.33 \mathrm{~dB} 29.39 \mathrm{~dB}$ & $28.88 \mathrm{~dB}$ & $28.46 \mathrm{~dB}$ & $28.98 \mathrm{~dB}$ \\
\hline Child-face & $31.29 \mathrm{~dB} 31.82 \mathrm{~dB}$ & $31.54 \mathrm{~dB}$ & $31.50 \mathrm{~dB}$ & $31.65 \mathrm{~dB}$ \\
\hline
\end{tabular}

The comparisons to the state-of-the-art methods on upsampling natural images are exemplified visually in Fig. 10 and shown in terms of PSNR in Table 1. It indicates that our upsamling method is able to preserve sharp edges and its performance is competitive both visually and in terms of PSNR. Moreover, the closer a natural image is to our piecewise smooth model, the better the performance of our method.

\section{CONCLUSIONS}

In this paper, we have proposed a scheme for sampling piecewise smooth signal. We show that the method proposed improves classical linear reconstruction results by making use of an additional nonlinear reconstruction method based on FRI theory. We also show that this approach can be used for resolution enhancement of discretetime piecewise smooth signals and images.
Fig. 9: Original high-resolution Peppers $(512 \times 512)$ and the lowresolution version $(128 \times 128)$.

\section{(b) low resolution version}




\section{REFERENCES}

[1] M. Unser, "Sampling-50 Years After Shannon," Proceedings of the IEEE, vol. 88, no. 4, pp. 569-587, 2000.

[2] M. Vetterli, P. Marziliano, and T. Blu, "Sampling Signals with Finite Rate of Innovation," IEEE Transactions on Signal Processing, vol. 50, no. 6, pp. 1417-1428, 2002.

[3] P. L. Dragotti, M. Vetterli, and T. Blu, "Sampling Moments and Reconstructing Signals of Finite Rate of Innovation: Shannon Meets Strang-Fix," IEEE Transactions on Signal Processing, vol. 55, no. 5, Part 1, pp. 1741-1757, 2007.

[4] T. Blu, P. L. Dragotti, M. Vetterli, P. Marziliano, and L. Coulot, "Sparse Sampling of Signal Innovations," Signal Processing Magazine, IEEE, vol. 25, no. 2, pp. 31-40, 2008.

[5] L. Baboulaz and P. L. Dragotti, "Exact Feature Extraction Using Finite Rate of Innovation Principles with an Application to Image Super-resolution," IEEE Transactions on Image Processing, vol. 18, no. 2, pp. 281-298, 2009.

[6] R. Tur, Y. C. Eldar, and Z. Friedman, "Innovation Rate Sampling of Pulse Streams with Application to Ultrasound Imaging," IEEE Transactions on Signal Processing, vol. 59, no. 4, pp. 1827-1842, 2011.

[7] J. A. Urigüen, Y. C. Eldar, P. L. Dragotti, and Z. Ben-Haim, "Sampling at the Rate of Innovation: Theory and Applications," Compressed Sensing: Theory and Applications, p. 148, 2012.

[8] X. Wei, T. Blu, and P. L. Dragotti, "Finite Rate of Innovation with Non-uniform Samples," in IEEE International Conference on Signal Processing, Communication and Computing (ICSPCC), August 2012, pp. 369 -372.

[9] J. A. Urigüen, T. Blu, and P. L. Dragotti, "FRI Sampling with Arbitrary Kernels," IEEE Transactions on Signal Processing, vol. 61, no. 21, pp. 5310-5323, 2013.

[10] M. Vetterli and J. Kovačević, Wavelets and Subband Coding, vol. 87, Prentice Hall PTR Englewood Cliffs, New Jersey, 1995.

[11] S. Mallat, A Wavelet Tour of Signal Processing, Academic Press, 1999.

[12] J. M. Bioucas-Dias and M. A. Figueiredo, "A New TwIST: Two-step Iterative Shrinkage/thresholding Algorithms for Image Restoration," IEEE Transactions on Image Processing, vol. 16, no. 12, pp. 2992-3004, 2007.

[13] N. Mueller, Y. Lu, and M. N. Do, "Image Interpolation Using Multiscale Geometric Representations," in Proc. SPIE Conf. on Electronic Imaging, San Jose, USA, 2007.

[14] R. Zeyde, M. Elad, and M. Protter, "On Single Image Scaleup Using Sparse-representations," in Curves and Surfaces, pp. 711-730. Springer, 2012. 